\title{
Antioxidant activity, total phenolics and flavonoids contents: should we ban in vitro screening methods?
}

Article

Accepted Version

Creative Commons: Attribution-Noncommercial-No Derivative Works 4.0

Granato, D., Shahidi, F., Wrolstad, R., Kilmartin, P., Melton, L. D., Hidalgo, F. J., Miyashita, K., Camp, J. v., Alasalvar, C., Ismail, A. B., Elmore, S., Birch, G. G., Charalampopoulos, D., Astley, S. B., Pegg, R., Zhou, P. and Finglas, P. (2018) Antioxidant activity, total phenolics and flavonoids contents: should we ban in vitro screening methods? Food Chemistry, 264. pp. 471-475. ISSN 0308-8146 doi:

https://doi.org/10.1016/j.foodchem.2018.04.012 Available at https://centaur.reading.ac.uk/77385/

It is advisable to refer to the publisher's version if you intend to cite from the work. See Guidance on citing.

Published version at: http://dx.doi.org/10.1016/j.foodchem.2018.04.012

To link to this article DOI: http://dx.doi.org/10.1016/j.foodchem.2018.04.012

Publisher: Elsevier

All outputs in CentAUR are protected by Intellectual Property Rights law, including copyright law. Copyright and IPR is retained by the creators or other copyright holders. Terms and conditions for use of this material are defined in the End User Agreement. 


\section{www.reading.ac.uk/centaur}

\section{CentAUR}

Central Archive at the University of Reading

Reading's research outputs online 


\section{Antioxidant activity, total phenolics and flavonoids contents: should we ban in vitro screening methods?}

Daniel Granato ${ }^{1}$, Fereidoon Shahidi ${ }^{2}$, Ronald Wrolstad ${ }^{3}$, Paul Finglas ${ }^{4}$,

Laurence D. Melton ${ }^{5}$, Francisco J. Hidalgo ${ }^{6}$, Kazuo Miyashita ${ }^{7}$, John van

Camp $^{8}$, Cesarettin Alasalvar ${ }^{9}$, Amin B. Ismail ${ }^{10}$, Stephen Elmore ${ }^{11}$, Gordon G.

Birch $^{11}$, Dimitris Charalampopoulos ${ }^{11}$, Sian B. Astley ${ }^{12}$, Sabine Baumgartner ${ }^{13}$

1Department of Food Engineering, State University of Ponta Grossa, Av. Carlos Cavalcanti, 4748, 84030-900, Ponta Grossa, Brazil. E-mail: dgranato@uepg.br, granatod@gmail.com

2Department of Biochemistry, Memorial University of Newfoundland, St. John's, NL A1B 3X9, Canada.

${ }^{3}$ Oregon State University, 100 Wiegand Hall, Corvallis, OR 97331, United States. ${ }^{4}$ Institute of Food Research, Norwich, United Kingdom.

${ }^{5}$ School of Chemical Sciences, University of Auckland, Private Bag 92019, Auckland 1142, New Zealand.

${ }^{6}$ Instituto de la Grasa, Consejo Superior de Investigaciones Científicas, Carretera de Utrera km 1, Campus Universitario - Edificio 46, 41013 Seville, Spain.

${ }^{7}$ Faculty of Fisheries Sciences, Hokkaido University, Hakodate 041-8611, Japan. ${ }^{8}$ Laboratory of Food Chemistry and Human Nutrition (nutriFOODchem), Department of Food Safety and Food Quality, Faculty of Bioscience Engineering, Ghent University, Coupure Links 653, B-9000 Ghent, Belgium. ${ }^{9}$ TÜBITAK Marmara Research Centre, Food Institute, Gebze-Kocaeli, Turkey. 
${ }^{10}$ Center for Quality Assurance (CQA), Universiti Putra Malaysia.

${ }^{11}$ Food and Nutritional Sciences, University of Reading, PO Box 217 Whiteknights, RG6 6AH, Reading, United Kingdom.

${ }^{12}$ EuroFIR AISBL, Brussels, Belgium.

${ }^{13}$ Universität für Bodenkultur Wien (BOKU), Vienna, Austria.

${ }^{14}$ University of Georgia, Athens, Georgia, United States.

${ }^{15}$ Jiangnan University, Wuxi, China.

\section{Abstract}

As many studies are disclosing the association between the ingestion of bioactive compounds and a decreased risk of noncommunicable diseases, the scientific community has shown much interest in these compounds. In addition, as bioactive compounds are regarded as reducing agents, hydrogen donors, singlet oxygen quenchers or metal chelators, the measurement of antioxidant activity by in vitro assays has become very popular in the last decades. Measuring the levels of total phenolics, flavonoids, and other (sub)classes using spectrophotometry represents a chemical index but chromatographic techniques are necessary to establish structure-activity. For bioactive purposes, in vivo models are recommended or, at very least, different methods that employ distinct mechanisms of action need to be used. In this regard, some comments were made concerning the in vitro screening methods that will help one to design future research studies on "bioactive compounds".

Keywords: Folin-Ciocalteu; antioxidants; bioavailability; colorimetric methods; functional properties; in vivo studies; HPLC. 


\section{Phenolic compounds as antioxidants}

Halliwell and Gutteridge (2007) state that "an antioxidant is a substance that, when present at a low concentration compared with that of an oxidizable substrate in the medium, inhibits oxidation of the substrate". In this classification, phenolic compounds, which are derived from the secondary metabolism of plants, can protect multiple organs from oxidation. Therefore, phenolic compounds are regarded as natural antioxidants.

Antioxidants are categorized based on their Function (free-radical scavengers, scavengers of non-radical oxidizing agents, compounds that inhibit the generation of oxidants, transition metal chelating agents, and compounds that are able to stimulate the production of endogenous antioxidant compounds); Polarity (water-soluble and liposoluble); Source: (exogenous or endogenous); Mechanism: Antioxidants can neutralize the deleterious action of reactive species of cell membranes mainly by three mechanisms: hydrogen atom transfer (HAT), electron transfer (ET), and the ability to chelate transition metals (Prior et al., 2005; Brewer, 2011). In this sense, the HAT mechanism measures the ability of an antioxidant $(\mathrm{AH})$ to quench free radicals (i.e., peroxyl radical - ROO*) by hydrogen donation stabilizing the peroxyl radical by resonance according to the Equation (1):

$$
\mathrm{AH}+\mathrm{ROO}^{\circ} \rightarrow \mathrm{ROOH}+\mathrm{A}^{\circ}
$$

The ET-based assays measure the ability of $\mathrm{AH}$ to transfer one electron to reduce free radicals, pro-oxidant metals and carbonyls, which are based on Equation (2) (Huang et al., 2005; Apak et al., 2013):

$$
\mathrm{ROO}+\mathrm{AH} \rightarrow \mathrm{ROO}^{\bullet}+\mathrm{AH}^{+} \leftrightarrow \mathrm{A}^{\cdot}+\mathrm{H}^{+}
$$


HAT assays include the oxygen radical absorbance capacity (ORAC), inhibition of lipoperoxidation, crocin bleaching assay, and $\beta$-carotene bleaching assay. Similarly, ET methods are composed of cupric-ion reducing antioxidant capacity (CUPRAC), Folin-Ciocalteu's phenol reagent reducing ability, scavenging effects in relation to 1,1-diphenyl-2-picrylhydrazyl (DPPH), and 2,2'azino-bis(3-ethylbenzothiazoline-6-sulphonic acid (ABTS), among others (Shahidi \& Zhong, 2015).

Some criticisms related to these in vitro chemical assays are based on the inexistence of such free radicals (DPPH/ABTS) in humans and the complexity of the mechanism of reaction. In addition, a high in vitro antioxidant activity cannot be translated into "treatment/cure" of illnesses. For instance, in the ferric reducing ability of plasma (FRAP) assay, as the reaction is performed at low $\mathrm{pH}$ values (3.6), much criticism is made on the translation of this method into in vivo effectiveness and, therefore, it can only be considered a screening method to have an idea of the antioxidant capacity of the sample (Schaich, Tian, \& Xie, 2015). Undoubtedly, as these chemical assays are low-cost, easy to perform, do not require ultra-sensitive equipment, they are used to assess both isolated compounds and extracts from complex food matrices.

The antioxidant activity of phenolic compounds has been studied using a wide variety of methods, including in vitro, ex vivo, and in vivo protocols. Usually, authors find a high degree of correlation between in vitro antioxidant activity and the total phenolic content and/or individual phenolics (Rodrigo et al., 2005). However, the association between in vitro and in vivo antioxidant methods is still debatable and the opinion of experts in the field is divided into the usefulness of such in vitro methods. 


\section{Should we ban in vitro screening method to assess the antioxidant} activity?

Several assays can be used to screen the in vitro antioxidant capacity of plant extracts, such as ferrous-ion chelating activity (Carter, 1971), copper chelating activity (Saiga, Tanabe, \& Nishimura, 2003), lipid peroxidation inhibition assay (Daker et al., 2008), CUPRAC (Apak et al., 2008), deoxyribose assay (Chen, Zhang, \& Xie, 2005), photoreduction of nitro blue tetrazolium assay (Chen, Zhang, \& Xie, 2005), superoxide dismutase mimetic activity (Naithani, Nair, \& Kakkar, 2006), total reducing capacity using a modified Folin-Ciocalteu assay (Berker et al., 2013), scavenging of hydrogen peroxide (Ruch, Cheng, \& Klaunig, 1989), and cell-based in vitro antioxidant activity (Kellett, Greenspan, \& Pegg, 2018). Excellent reviews on several chemical in vitro and cellular-based assays to assess the antioxidant activity can be found elsewhere (Alves et al., 2010; Niki, 2010; López-Alarcón \& Denicól, 2013; Shahidi \& Zhong, 2015). Without a doubt, the most frequently used methods rely on the use of DPPH, ABTS, FRAP, and ORAC assays (Halliwell, 2012; Schaich, Tian, \& Xie, 2015). These methods have many pros and cons, as any other analytical method, but when the antioxidant activity is evaluated, these methods have particularities in relation to the mechanism of action of the $\mathrm{AH}$, the type of target (i.e., $\mathrm{H}_{2} \mathrm{O}_{2}$ or DPPH radical), reactional $\mathrm{pH}$, reaction time and temperature, and the use of a standard to build an analytical curve that is used to give a quantitative result in terms of antioxidant activity (Forman et al., 2014). Therefore, no single in vitro antioxidant activity assay will reflect the "total" antioxidant effect (Apak et al., 2013; Berker et al., 2013). 
Recently, Harnly (2017) stated that studies regarding the measurement of in vitro antioxidant activity and total phenolic content using the Folin-Ciocalteu reagent is not appropriate. The reasons are:

1. There is currently no accepted standard mechanism or method to measure the antioxidant activity;

2. Only state-of-the-art techniques to identify antioxidants (i.e., flavonoids) should be used in scientific research;

3. Results of a method X (i.e., FRAP) are (usually) not comparable with data obtained using the method $Y$ (i.e., DPPH) or even between laboratories; and 4. Antioxidant is a marketing term of questionable health and analytical value as epidemiological studies are inconsistent.

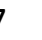

In this regard, it is unquestionable that "state-of-the-art" techniques, such as liquid chromatography-mass spectroscopy (LC-MS), to identify and quantify phenolic compounds in foods, beverages, and herbal extracts have high accuracy and precision. However, screening spectrophotometric methods should also be used to characterize these materials and have an idea of the total content of phenolic compounds in the matrix (Granato, Santos, Maciel, \& Nunes, 2016). Halliwell (2012) stated that "the consumption of mega-doses of antioxidants (i.e., pills) have also generally failed to prevent human disease, in part because they do not decrease oxidative damage in vivo". Individuality (i.e., genetics, gender, and body mass index) and life habits (i.e., exercising, drugs/alcohol abuse, and smoking) also play an important role in the oxidative status of humans. Although some studies show discrepancies and inconsistencies to show a clear association between consumption of phenolic 
151

compounds and increase of the antioxidant status in humans (Frankel \& German, 2006; Saldanha et al., 2016), the search for antioxidants should continue and any allegation on functionality should be supported by preclinical, clinical, and epidemiological studies.

As well known, in vitro antioxidant methods and the estimation of total phenolic content using colorimetric assays can be used not only to have an idea of the beneficial effects of the food/extract. For quality control of natural products (Guo, Sun, Yu, \& Qi, 2017; Lv, Zhang, Shi, \& Lin, 2017), the antioxidant activity measured by in vitro methods are very useful as a fingerprint of reference materials that can be used for comparison purposes with commercial samples. Therefore, trends are generally very useful for comparative purposes of samples of the same material. In food technology, in vitro antioxidant assays together with the total phenolic content may be of importance to assess the best cutting styles of fruits ( $\mathrm{Li}$ et al., 2017). These examples illustrate the usefulness of in vitro methodologies that can be applied in the routine quality control programs of food companies worldwide. Without a doubt, interferences in these nonselective methodologies exist and this fact is well demonstrated when comparing highperformance liquid chromatography (HPLC) results with total contents of phenolic compounds. Nevertheless, we need to have something in mind: one cannot rule out the usefulness of in vitro results despite their imperfect nature.

To date, Williams, Soencer, and Rice-Evans (2004) stated that "phenolic compounds may exert modulatory actions in cells through actions at protein kinase and lipid kinase signaling pathways A clear understanding of the mechanisms of action of flavonoids, either as antioxidants or modulators of cell signaling, and the influence of their metabolism on these properties are key to 
176 the evaluation of these potent biomolecules as anticancer agents, 177 cardioprotectants, and inhibitors of neurodegeneration". In addition, Alam, Bristi, \& Rafiquzzaman (2013) stated that "antioxidants may be of great benefit in improving the quality of life by preventing or postponing the onset of noncommunicable diseases".

In recent studies, the antioxidant activity of bioactive compounds measured by in vitro and in vivo models are associated in a way that, depending on the biomarker used to assess the oxidative stress, interesting conclusions with practical applications arise (Macedo et al., 2013; Yan, Chen, \& Zheng, 2017; Sun et al., 2017; Villa-Hernández et al., 2017; Aouachria et al., 2017; Naeimi \& Alizadeh, 2017; Donado-Pestana et al., 2018). Obviously, there is a need to demonstrate the mechanistic approach behind the antioxidant activity of polyphenols in vivo. Animal models (i.e., rat, mouse, rabbit, and dog) and human studies (i.e., preclinical and randomized double-blind placebo-controlled clinical trials) are more appropriate but also more expensive, complex, and timeconsuming compared to chemical and cellular-based methods (Thompson, Pederick, Singh, \& Santhakumar, 2017). The assessment of in vivo antioxidant activity should include the measurement the activity of endogenous enzymes and antioxidant gene expression compared to a placebo, for instance. The could be absorbed, there is sometimes an insufficient concentration of the antioxidants in target tissues for the activity to be the prevalent protective mechanism (Huang et al., 2017). 
Another point of consideration is as follows: what is measured in the food

202 is not fully representative for what is active in humans. As well stressed by Espín,

203 González-Sarrías, and Tomás-Barberán (2017) and Granado-Lorencio, Blanco-

204 Navarro, Pérez-Sacristán, and Hernández-Álvarez (2017), “the type and quantity

205 of the carotenoid/phenolic compounds metabolites produced in humans depend

206 on the gut microbiota composition and function. The beneficial effect biological

207 upon carotenoid/polyphenols intervention varies considerably and the chronic

208 use of large doses may lead to saturation effects and the loss of linearity in the

209 response.Therefore, the final health effects of dietary polyphenols/carotenoids

210 depend on the gut microbiota composition". As the microbiota of each individual

211 is unique, we cannot assume "functionality" based only on in vitro tests.

\section{Finals remarks and conclusions}

As a conclusion of this viewpoint, although there will be divergent opinions in the scientific community based on thousands of studies available, we cannot close our eyes to dietary antioxidants and ignore some in vitro screening methods (i.e., total phenolic/total flavonoids contents and antioxidant activity measurements) as low-cost, high-throughput tools to discover potential antioxidant sources for human consumption.

In a perspective, manuscripts on antioxidant properties based solely on colorimetric methods (including the Folin-Ciocalteu assay) will become unacceptable in Food Chemistry from now on. Authors are encouraged to assay bioactive compounds using chromatographic techniques (i.e., HPLC/LC-MS) and, preferably, there must be some biological tests using cell lines or simulated digestion, or at the very least, measurement of bioactivity (i.e., antioxidant effect) 
using multiple assays that employ different mechanisms of action (i.e., HAT, ET, and metal chelation property).

\section{References}

Alam, M. D., Bristi, N. J., \& Rafiquzzman, M. (2013). Review on in vivo and in vitro methods evaluation of antioxidant activity. Saudi Pharmaceutical Journal, $21,143-152$.

Alves, C. Q., David, J. M., David, J. P., Bahia, M. V., \& Aguiar, R. M. (2010). Methods for determination of in vitro antioxidant activity for extracts and organic compounds. Química Nova, 33, 2202-2210.

Aouachria, S., Boumerfeg, S., Benslama, A., Benbacha, F., Guemmez, T., Khennouf, S., Arrar, L., \& Baghiani, A. (2017). Acute, sub-acute toxicity and antioxidant activities (in vitro and in vivo) of Reichardia picroide crude extract. Journal of Ethnopharmacology, 208, 105-116.

Apak, R., Gorinstein, S., Böhm, V., Schaich, K. M., Özyürek, M., \& Güçlü, K. (2013). Methods of measurement and evaluation of natural antioxidant capacity/activity (IUPAC Technical Report). Pure and Applied Chemistry, 85(5), 957-998.

Apak, R., Guclu, K., Ozyurek, M., \& Celik, S. E. (2008). Mechanism of antioxidant capacity assays and the CUPRAC (cupric ion reducing antioxidant capacity) assay. Microchimica Acta, 160(4), 413-419.

Berker, K. I., Olgun, F. A. O., Ozyurt, D., Demirata, B., \& Apak, R. (2013). Modified Folin-Ciocalteu antioxidant capacity assay for measuring lipophilic antioxidants. Journal of Agricultural and Food Chemistry, 61, 4783-4791. 
250 Brewer, M. S. (2011). Natural antioxidants: sources, compounds, mechanisms of

251 action, and potential applications. Comprehensive Reviews in Food Science and 252 Food Safety, 10, 221-247.

253 Carter, P. (1971). Spectrophotometric determination of serum iron at the 254 submicrogram level with a new reagent (ferrozine). Analytical Biochemistry, $255 \quad 40(2), 450-458$.

256 Chen, H., Zhang, M., \& Xie, B. (2005). Components and antioxidant activity of 257 polysaccharide conjugate from green tea. Food Chemistry, 90, 17-21.

258 Daker, M., Abdullah, N., Vikineswary, S., Goh, P. C., \& Kuppusamy, U. R. (2008). 259 Antioxidant from maize and maize fermented by Marasmiellus sp. as stabiliser of 260 lipid-rich foods. Food Chemistry, 107, 1092-1098.

261 Donado-Pestana, C. M., dos Santos-Donado, P. R., Daza, L. D., Belchior, T., 262 Festuccia, W. T., \& Genovese, M. I. (2018). Cagaita fruit (Eugenia dysenterica 263 DC.) and obesity: Role of polyphenols on already established obesity. Food 264 Research International, 103, 40-47.

265 Espín, J. C., González-Sarrías, A., \& Tomás-Barberán, F. A. (2017). The gut 266 microbiota: A key factor in the therapeutic effects of (poly)phenols. Biochemical 267 Pharmacology, 139, 82-93.

268 Forman, H. J., Davies, K. J. A., \& Ursini, F. (2014). How do nutritional antioxidants 269 really work: Nucleophilic tone and para-hormesis versus free radical scavenging 270 in vivo. Free Radical in Biology \& Medicine, 66, 24-35.

271 Frankel, E. N., \& German, J. B. (2006). Antioxidants in foods and health: problems and fallacies in the field. Journal of the Science of Food and Agriculture, 86, 1999-2001. 
274 Granado-Lorencio, F., Blanco-Navarro, I., Pérez-Sacristán, B., \& Hernández-

275 Álvarez, E. (2017). Biomarkers of carotenoid bioavailability. Food Research 276 International, 99(2), 902-916.

277 Granato, D., Santos, J. S., Maciel, L. G., \& Nunes, D. S. (2016). Chemical 278 perspective and criticism on selected analytical methods used to estimate the 279 total content of phenolic compounds in food matrices. Trends in Analytical 280 Chemistry, 80, 266-279.

281 Guo, Y., Sun, L., Yu, B., \& Qi, J. (2017). An integrated antioxidant activity 282 fingerprint for commercial teas based on their capacities to scavenge reactive 283 oxygen species. Food Chemistry, 237, 645-653.

284 Halliwell, B. (2012). Free radicals and antioxidants: updating a personal view. 285 Nutrition Review, 70(5), 257-265.

286 Halliwell, B. and Gutteridge, J. M. C. (2007). Free Radicals in Biology and 287 Medicine. $4^{\text {th }}$ edition. Oxford: Oxford University Press, 888p.

288 Harnly, J. (2017). Antioxidant methods. Journal of Food Composition and 289 Analysis, in press.

290 Huang, D., Ou, B. and Prior, R. L. (2005). The chemistry behind antioxidant 291 capacity assay. Journal of Agricultural and Food Chemistry, 53, 1841-1856.

292 Huang, S., Ma, Y., Zhang, C., Cai, S., \& Pang, M. (2017). Bioaccessibility and 293 antioxidant activity of phenolics in native and fermented Prinsepia utilis Royle seed during a simulated gastrointestinal digestion in vitro. Journal of Functional 295 Foods, 37, 354-362.

296 Kellett, M. E., Greenspan, P., \& Pegg, R. B. (2018). Modification of the Cellular 297 Antioxidant Activity (CAA) assay to study phenolic antioxidants in a Caco-2 cell 298 line. Food Chemistry, In press 
299 Li, X., Long, Q., Gao, F., Han, C., Jin, P., \& Zheng, Y. (2017). Effect of cutting styles on quality and antioxidant activity in fresh-cut pitaya fruit. Postharvest Biology and Technology, 124, 1-7.

López-Alarcón, C., \& Denicol, A. (2013). Evaluating the antioxidant capacity of natural products. A review on chemical and cellular-based assays. Analytica Chimica Acta, 763, 1-10.

Lv, H., Zhang, Y., Shi, J., \& Lin, Z. (2017). Phytochemical profiles and antioxidant activities of Chinese dark teas obtained by different processing technologies. Food Research International, 100, 486-493.

Macedo, L. F. L., Rogero, M. M., Guimarães, J. P., Granato, D., Lobato, L. P., \& Castro, I. A. (2013). Effect of red wines with different in vitro antioxidant activity on oxidative stress of high-fat diet rats. Food Chemistry, 137, 122-129. Naeimi, A. F., \& Alizadeh, A. (2017). Antioxidant properties of the flavonoid fisetin: An updated review of in vivo and in vitro studies. Trends in Food Science and Technology, 70, 34-44.

Naithani, V., Nair, S. \& Kakkar, P. (2006). Decline in antioxidant capacity of Indian herbal teas during storage and its relation to phenolic content. Food Research International, 39, 176-181.

317 Niki, E. (2010). Assessment of antioxidant capacity in vitro and in vivo. Free Radical Biology and Medicine, 49(4), 503-215.

319 Prior, R. L., Wu, X. \& Schaich, K. (2005). Standard methods for the determination 320 of antioxidant capacity and phenolics in foods and dietary supplements. Journal of Agricultural and Food Chemistry, 53, 4290-4302. 
322 Rodrigo, R., Castillo, R., Carrasco, R., Huerta, P., \& Moreno, M. (2005).

323 Diminution of tissue lipid peroxidation in rats is related to the in vitro antioxidant 324 capacity of wine. Life Sciences, 76, 889-900.

325 Ruch, R. J., Cheng, S. J., \& Klaunig, J. E. (1989). Prevention of cytotoxicity and 326 inhibition of intracellular communication by antioxidant catechins isolated from 327 Chinese green tea. Carcinogenesis, 10, 1003-1008.

Saiga, A. I., Tanabe, S., \& Nishimura, T. (2003). Antioxidant activity of peptides obtained from porcine myofibrillar proteins by protease treatment. Journal of Agricultural and Food Chemistry, 51(12), 3661-3667.

Saldanha, J. F., Leal, V. O., Rizzetto, F., Grimmer, G. H., Ribeiro-Alves, M.,

Delaprene, J. B., Carraro-Eduardo, J. C. \& Mafra, D. (2016). Effects of resveratrol supplementation in Nrf2 and Nf-kb expressions in nondialyzed chronic kidney disease patients: a randomized, double-blind, placebo-controlled, crossover clinical trial. Journal of Renal Nutrition, 26(6), 401-406

Schaich, K. M., Tian, X., \& Xie, J. (2015). Hurdles and pitfalls in measuring antioxidant efficacy: A critical evaluation of ABTS, DPPH, and ORAC assays. Journal of Functional Foods, 14, 111-125.

Shahidi, F., \& Zhong, Y. (2015). Measurement of antioxidant activity. Journal of Functional Foods, 18, 757-781. in vivo antioxidant activity of polyphenols extracted from black highland barley. Food Chemistry, 194, 1003-1012.

344 Sun, Y., Tao, X., Men, X., Xu, Z., \& Wang, T. (2017). In vitro and in vivo antioxidant activities of three major polyphenolic compounds in pomegranate 
346 peel: Ellagic acid, punicalin, and punicalagin. Journal of Integrative Agriculture, 347 16(8), 1808-1818.

348 Thompson, K., Pederick, W., Singh, I., \& Santhakumar, A. B. (2017). Anthocyanin 349 supplementation in alleviating thrombogenesis in overweight and obese 350 population: A randomized, double-blind, placebo-controlled study. Journal of 351 Functional Foods, 32, 131-138.

352 Villa-Hernández, J. M., Mendoza-Cardoso, G., Mendoza-Espinoza, J. A., Vela353 Hinojosa, C., León-Sánchez, F. D., Rivera-Cabrera, F., Alia-Tejacal, I., \& Pérez354 Flores, L. J. Antioxidant capacity in vitro and in vivo of various ecotypes of 355 Mexican plum (Spondias purpurea L.). Journal of Food Science, in press.

356 Williams, R. J., Soencer, J. P. E., \& Rice-Evans, C. (2004). Flavonoids: 357 antioxidants or signaling molecules. Free Radical Biology \& Medicine, 36, 838358849.

359 Yan, F., Chen, X., \& Zheng, X. (2017). Protective effect of mulberry fruit 360 anthocyanin on human hepatocyte cells (LO2) and Caenorhabditis elegans under 361 hyperglycemic conditions. Food Research International, 102, 213-224. 